\title{
Spin currents, spin torques, and the concept of spin superfluidity
}

\author{
Andreas Rückriegel and Peter Kopietz \\ Institut für Theoretische Physik, Universität Frankfurt, \\ Max-von-Laue Strasse 1, 60438 Frankfurt, Germany
}

(Dated: January 19, 2017)

\begin{abstract}
In magnets with non-collinear spin configuration the expectation value of the conventionally defined spin current operator contains a contribution which renormalizes an external magnetic field and hence affects only the precessional motion of the spin polarization. This term, which has been named angular spin current by Sun and Xie [Phys. Rev B 72, 245305 (2005)], does not describe the translational motion of magnetic moments. We give a prescription how to separate these two types of spin transport and show that the translational movement of the spin is always polarized along the direction of the local magnetization. We also show that at vanishing temperature the classical magnetic order parameter in magnetic insulators cannot carry a translational spin current, and elucidate how this affects the interpretation of spin supercurrents.
\end{abstract}

\section{INTRODUCTION}

The notion of spin currents describing the motion of magnetic moments associated with the spins of the electrons in solids is of central importance in the field of spintronics where one tries to use the spin degree of freedom to store and process information. Unfortunately, in systems lacking spin-rotational invariance (which can be broken by an external magnetic field or by relativistic effects such as spin-orbit coupling or dipole-dipole interactions) the proper definition of the quantum mechanical operator representing the spin current is ambiguous, because the magnetization does not satisfy a local conservation law. In the past decade several authors have proposed resolutions of this ambiguity, $\frac{1-16}{16}$ but a generally accepted agreement on the correct definition of the spin current operator in systems without spin conservation has not been found.

The purpose of this work is show that the distinction between translational and angular spin currents proposed by Sun and $\mathrm{Xic}^{8}$ leads to a simple and unique definition of the concept of spin transport in condensed matter systems. Sun and $\mathrm{Xie}^{8}$ pointed out that spin currents describe moving magnetic dipoles, and that generally the transport of any vector can be decomposed into a translational part characterized by some velocity $\boldsymbol{v}(\boldsymbol{r})$ and an angular part described by some angular velocity $\boldsymbol{\omega}(\boldsymbol{r})$, see Fig. 1. In the context of spin transport Sun and $\mathrm{Xie}^{8}$ called the latter contribution the angular spin current, although this can be also viewed as the spin torque discussed earlier by Culcer et al. [6]. We will explicitly show below that the equation of motion of a magnetic moment $\boldsymbol{m}_{i}$ at lattice site $\boldsymbol{R}_{i}$ with magnitude $m_{i}=\left|\boldsymbol{m}_{i}\right|$ and polarization $\hat{\boldsymbol{m}}_{i}=\boldsymbol{m}_{i} /\left|\boldsymbol{m}_{i}\right|$ can be decomposed into a translational part

$$
\partial_{t} m_{i}+\sum_{j}\left\langle I_{i \rightarrow j}^{\|}\right\rangle=0
$$

that corresponds to the physical movement of magnetic dipole moments, and an angular part

$$
\partial_{t} \hat{\boldsymbol{m}}_{i}=\boldsymbol{\omega}_{i} \times \hat{\boldsymbol{m}}_{i}
$$

(a)

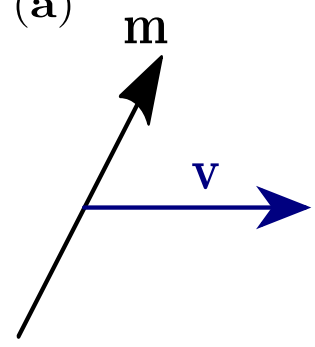

(b)

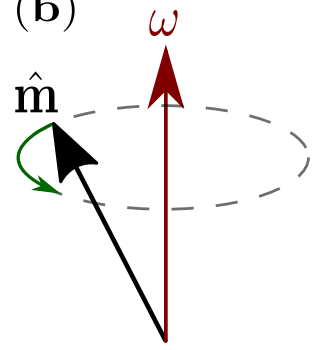

FIG. 1. (Color online) The two kinds of spin transport. (a) Translational motion of the magnetic moment $\boldsymbol{m}$ with a velocity $\boldsymbol{v}$, corresponding to the physical movement of a magnetic moment with magnitude $|\boldsymbol{m}|$. (b) Precessional motion of the magnetic polarization $\hat{\boldsymbol{m}}=\boldsymbol{m} /|\boldsymbol{m}|$ with a frequency $\boldsymbol{\omega}$. The latter motion is purely angular and leaves the magnitude $|\boldsymbol{m}|$ of the magnetic moment invariant.

that describes the precessional motion of the magnetization. We will provide expressions for the translational spin current operator $I_{i \rightarrow j}^{\|}$and the precession frequency $\boldsymbol{\omega}_{i}$ valid for itinerant as well as localized systems. Although the explicit construction of this decomposition is very simple, it entails profound physical consequences: Since only the translational movement of magnetization corresponds to the physical displacement of magnetic moments, in equilibrium only stationary translational spin currents can generate an electrical field ${ }^{17}$ Angular spin currents on the other hand only transport the polarization, hence a stationary angular spin current is simply an inhomogeneous configuration of the local magnetic order and does not create an electrical field. Furthermore, we also show that in generic magnetic insulators the classical magnetic order cannot support translational spin transport at vanishing temperature; incoherent thermal or quantum fluctuations are mandatory for the physical transport of magnetization in these systems. This also implies that spin superfluidity in magnetic insulators 18 |26 must be angular spin transport that can be visualized as transporting the spin polarization, but does not corre- 
spond to the physical movement of magnetic moments.

The remainder of this work is organized as follows: In Sec. [II we will derive general expressions for the operators corresponding to the translational spin current and to the precession frequency of the magnetization, first for itinerant systems and then for localized magnetic moments. We proceed to illustrate the usefulness of the decoupling procedure in Sec. III], where we discuss spin superfluidity in easy-plane ferromagnets and persistent spin currents in Heisenberg rings. Finally, in Sec. [V] we present our conclusions. The Appendix contains some additional details of the self-consistent spin-wave expansion we employ to describe easy-plane ferromagnets.

\section{SEPARATING TRANSLATIONAL FROM ANGULAR SPIN TRANSPORT}

In this section, we explicitly show how translational and angular spin transport can be defined on the operator level.

\section{A. Itinerant electrons}

To construct the proper quantum mechanical definition of the translational spin transport operator let us consider a lattice model describing electrons with spindependent hopping $t_{i j}^{\sigma \sigma^{\prime}}$ in an inhomogeneous magnetic field $\boldsymbol{h}_{i}$. The second quantized Hamiltonian of our model is

$$
\mathcal{H}=\sum_{i j \sigma \sigma^{\prime}} t_{i j}^{\sigma \sigma^{\prime}} c_{i \sigma}^{\dagger} c_{j \sigma^{\prime}}-\sum_{i} \boldsymbol{h}_{i} \cdot \boldsymbol{s}_{i}+\mathcal{U}
$$

where $\mathcal{U}$ is some spin-rotationally invariant interaction, $c_{i \sigma}$ annihilates a fermion with spin-projection $\sigma$ at lattice site $\boldsymbol{R}_{i}$, and the itinerant spin operators are defined by

$$
\boldsymbol{s}_{i}=\frac{1}{2} c_{i}^{\dagger} \boldsymbol{\sigma} c_{i}, \quad c_{i}=\left(\begin{array}{c}
c_{i \uparrow} \\
c_{i \downarrow}
\end{array}\right) .
$$

Here $\boldsymbol{\sigma}$ is the vector of Pauli matrices. The spindependent hopping energies $t_{i j}^{\sigma \sigma^{\prime}}$ are of the form

$$
t_{i j}^{\sigma \sigma^{\prime}}=t_{i j} \delta_{\sigma \sigma^{\prime}}+i\left(\boldsymbol{\lambda}_{i j} \cdot \boldsymbol{\sigma}\right)_{\sigma \sigma^{\prime}}
$$

where the vectors $\boldsymbol{\lambda}_{i j}$ are proportional to the strength of the spin-orbit coupling. The hermiticity of the Hamiltonian implies the symmetries $t_{i j}=t_{j i}^{*}$ and $\boldsymbol{\lambda}_{i j}=$ $-\boldsymbol{\lambda}_{j i}^{*}$. Using the canonical anticommutation relations $\left\{c_{i \sigma}, c_{j \sigma^{\prime}}^{\dagger}\right\}=\delta_{i j} \delta_{\sigma \sigma^{\prime}}$ and the fact that the interaction is spin-rotationally invariant, $\left[\boldsymbol{s}_{i}, \mathcal{U}\right]=0$, we obtain the Heisenberg equation of motion for the itinerant spins,

$$
\frac{\partial \boldsymbol{s}_{i}}{\partial t}+\sum_{j} \boldsymbol{I}_{i \rightarrow j}=-\boldsymbol{h}_{i} \times \boldsymbol{s}_{i}
$$

where we have defined the operator

$$
\begin{aligned}
\boldsymbol{I}_{i \rightarrow j}= & -\frac{1}{2 i}\left(t_{i j} c_{i}^{\dagger} \boldsymbol{\sigma} c_{j}-t_{i j}^{*} c_{j}^{\dagger} \boldsymbol{\sigma} c_{i}\right) \\
& -\frac{1}{2}\left(\boldsymbol{\lambda}_{i j} c_{i}^{\dagger} c_{j}+\boldsymbol{\lambda}_{i j}^{*} c_{j}^{\dagger} c_{i}\right) \\
& -\frac{1}{2 i}\left(c_{i}^{\dagger}\left(\boldsymbol{\sigma} \times \boldsymbol{\lambda}_{i j}\right) c_{j}-c_{j}^{\dagger}\left(\boldsymbol{\sigma} \times \boldsymbol{\lambda}_{i j}^{*}\right) c_{i}\right) .
\end{aligned}
$$

It is tempting to associate this operator with the spin current describing the transport of spin from lattice site $\boldsymbol{R}_{i}$ to lattice site $\boldsymbol{R}_{j}$. It turns out, however, that a certain part of this operator simply renormalizes the external magnetic field and therefore cannot be associated with translational spin transport. To isolate this contribution and identify the angular part which renormalizes the precessional motion of the spins, we take the quantum mechanical expectation value of both sides of the equation of motion (6) and obtain a formally exact equation of motion for the magnetic moments $\boldsymbol{m}_{i}(t)=\left\langle\boldsymbol{s}_{i}(t)\right\rangle$,

$$
\partial_{t} \boldsymbol{m}_{i}+\boldsymbol{T}_{i}=-\boldsymbol{h}_{i} \times \boldsymbol{m}_{i},
$$

where the spin torque is defined by

$$
\boldsymbol{T}_{i}=\sum_{j}\left\langle\boldsymbol{I}_{i \rightarrow j}\right\rangle .
$$

To identify the contribution responsible for translational spin transport, we further decompose the vector $\boldsymbol{T}_{i}$ into a longitudinal and a transverse part,

$$
\boldsymbol{T}_{i}=T_{i}^{\|} \hat{\boldsymbol{m}}_{i}+\boldsymbol{T}_{i}^{\perp},
$$

where $\hat{\boldsymbol{m}}_{i}=\boldsymbol{m}_{i} /\left|\boldsymbol{m}_{i}\right|$ is the local spin polarization and

$$
\begin{aligned}
T_{i}^{\|} & =\sum_{j} \hat{\boldsymbol{m}}_{i} \cdot\left\langle\boldsymbol{I}_{i \rightarrow j}\right\rangle, \\
\boldsymbol{T}_{i}^{\perp} & =\boldsymbol{T}_{i}-\left(\boldsymbol{T}_{i} \cdot \hat{\boldsymbol{m}}_{i}\right) \hat{\boldsymbol{m}}_{i} .
\end{aligned}
$$

Writing

$$
\boldsymbol{T}_{i}^{\perp}=\left(\hat{\boldsymbol{m}}_{i} \times \boldsymbol{T}_{i}\right) \times \hat{\boldsymbol{m}}_{i}=\delta \boldsymbol{h}_{i}^{\perp} \times \boldsymbol{m}_{i},
$$

where

$$
\delta \boldsymbol{h}_{i}^{\perp}=\frac{\hat{\boldsymbol{m}}_{i} \times \boldsymbol{T}_{i}}{\left|\boldsymbol{m}_{i}\right|}=\sum_{j} \frac{\hat{\boldsymbol{m}}_{i}}{\left|\boldsymbol{m}_{i}\right|} \times\left\langle\boldsymbol{I}_{i \rightarrow j}\right\rangle
$$

is the induced magnetic field perpendicular to the direction of $\boldsymbol{m}_{i}$, we see that the transverse part $\boldsymbol{T}_{i}^{\perp}$ renormalizes the external magnetic field. The total angular frequency relevant for the precessional motion of the magnetic moments is

$$
\boldsymbol{\omega}_{i}=-\boldsymbol{h}_{i}-\delta \boldsymbol{h}_{i}^{\perp} .
$$

The term $-\delta \boldsymbol{h}_{i}^{\perp} \times \boldsymbol{m}_{i}$ can be called angular spin current ${ }^{8}$ or spin torque ${ }^{6}$ and should be added to the external torque $-\boldsymbol{h}_{i} \times \boldsymbol{m}_{i}$ acting on the magnetic moments. The 
expectation value of the equation of motion (8) can now be written as

$$
\partial_{t} \boldsymbol{m}_{i}+\hat{\boldsymbol{m}}_{i} T_{i}^{\|}=\boldsymbol{\omega}_{i} \times \boldsymbol{m}_{i}
$$

From this expression it is easy to show that the spin torque does not contribute to the time-evolution of the magnitude $m_{i}=\left|\boldsymbol{m}_{i}\right|$ of the magnetic moments, which satisfies the equation of motion

$$
\partial_{t} m_{i}+\sum_{j} \hat{\boldsymbol{m}}_{i} \cdot\left\langle\boldsymbol{I}_{i \rightarrow j}\right\rangle=0 .
$$

In contrast, the precessional motion of the spin polarization is governed solely by the spin torque,

$$
\partial_{t} \hat{\boldsymbol{m}}_{i}=\boldsymbol{\omega}_{i} \times \hat{\boldsymbol{m}}_{i}
$$

In summary, the renormalized precession frequency associated with the angular spin current is

$$
\boldsymbol{\omega}_{i}=-\boldsymbol{h}_{i}-\sum_{j} \frac{\hat{\boldsymbol{m}}_{i}}{\left|\boldsymbol{m}_{i}\right|} \times\left\langle\boldsymbol{I}_{i \rightarrow j}\right\rangle,
$$

while the operator representing the translational spin current is

$$
I_{i \rightarrow j}^{\|}=\hat{\boldsymbol{m}}_{i} \cdot \boldsymbol{I}_{i \rightarrow j}
$$

Note that time-dependent changes in the length of the magnetization are always accompanied by translational spin transport. On the other hand, stationary translational spin currents are also possible if the length of the magnetization is constant.

\section{B. Localized spins}

The above expressions have been derived for a lattice model for itinerant electrons. It is instructive to work out the explicit form of the rotation vector $\boldsymbol{\omega}_{i}$ and the translational spin current operator $I_{i \rightarrow j}^{\|}$for a localized spin model containing only the spin degrees of freedom. For simplicity, let us specify the interaction to the onsite Hubbard interaction $\mathcal{U}=U \sum_{i} n_{i \uparrow} n_{i \downarrow}$, where $n_{i \sigma}=$ $c_{i \sigma}^{\dagger} c_{i \sigma}$. Assuming $U \gg\left|t_{i j}^{\sigma \sigma^{\prime}}\right|$ and a half-filled lattice, we can use a canonical transformation ${ }^{27}$ to derive from Eq. (3) an effective Hamiltonian involving only spin 1/2 operators $\boldsymbol{S}_{i}$ acting on the reduced Hilbert space of singly occupied lattice sites. The effective spin Hamiltonian can be written as 28

$$
\begin{aligned}
\mathcal{H}^{\text {spin }}= & -\frac{1}{2 U} \sum_{i j}\left(\left|t_{i j}\right|^{2}+\left|\boldsymbol{\lambda}_{i j}\right|^{2}\right) \\
& +\frac{1}{2} \sum_{i j} \sum_{\alpha \beta} \mathbb{K}_{i j}^{\alpha \beta} S_{i}^{\alpha} S_{j}^{\beta}-\sum_{i} \boldsymbol{h}_{i} \cdot \boldsymbol{S}_{i},
\end{aligned}
$$

where the spin-spin interaction tensor has three contributions,

$$
\mathbb{K}_{i j}^{\alpha \beta}=\delta_{\alpha \beta} J_{i j}+\epsilon_{\alpha \beta \gamma} D_{i j}^{\gamma}+\Gamma_{i j}^{\alpha \beta}
$$

Here the isotropic exchange coupling $J_{i j}$ and the antisymmetric Dzyaloshinskii-Moriya vector $\boldsymbol{D}_{i j}$ are given by

$$
\begin{aligned}
J_{i j} & =\frac{4}{U}\left(\left|t_{i j}\right|^{2}-\left|\boldsymbol{\lambda}_{i j}\right|^{2}\right), \\
\boldsymbol{D}_{i j} & =-\frac{8}{U} \operatorname{Re}\left[t_{i j} \boldsymbol{\lambda}_{j i}\right]=\frac{8}{U} \operatorname{Re}\left[t_{i j} \boldsymbol{\lambda}_{i j}^{*}\right],
\end{aligned}
$$

while $\Gamma_{i j}^{\alpha \beta}$ is a symmetric tensor in spin space with matrix elements

$$
\Gamma_{i j}^{\alpha \beta}=-\frac{4}{U}\left(\lambda_{i j}^{\alpha} \lambda_{j i}^{\beta}+\lambda_{i j}^{\beta} \lambda_{j i}^{\alpha}\right)=\frac{8}{U} \operatorname{Re}\left[\lambda_{i j}^{\alpha}\left(\lambda_{i j}^{\beta}\right)^{*}\right] .
$$

The Heisenberg equation of motion can be written as

$$
\partial_{t} \boldsymbol{S}_{i}+\sum_{j} \boldsymbol{I}_{i \rightarrow j}^{\mathrm{spin}}=-\boldsymbol{h}_{i} \times \boldsymbol{S}_{i}
$$

where the operator

$$
\boldsymbol{I}_{i \rightarrow j}^{\text {spin }}=\boldsymbol{S}_{i} \times \mathbb{K}_{i j} \boldsymbol{S}_{j}
$$

is the strong coupling limit of the operator $\boldsymbol{I}_{i \rightarrow j}$ defined in Eq. (7) in the reduced spin Hilbert space. Here $\mathbb{K}_{i j}$ is a tensor in spin space with matrix elements given by Eq. 22). Alternatively, Eq. 27) can be obtained directly from Eq. (7) via a canonical transformation.27 With the substitution $\boldsymbol{I}_{i \rightarrow j} \rightarrow \boldsymbol{I}_{i \rightarrow j}^{\text {spin }}$ the expressions 19 and 20 for the local precession frequency and the longitudinal spin transport operator remain valid, so that we obtain

$$
\begin{aligned}
\boldsymbol{\omega}_{i} & =-\boldsymbol{h}_{i}-\sum_{j} \frac{\hat{\boldsymbol{m}}_{i}}{\left|\boldsymbol{m}_{i}\right|} \times\left\langle\boldsymbol{S}_{i} \times \mathbb{K}_{i j} \boldsymbol{S}_{j}\right\rangle, \\
\left\langle I_{i \rightarrow j}^{\|}\right\rangle & =\hat{\boldsymbol{m}}_{i} \cdot\left\langle\boldsymbol{S}_{i} \times \mathbb{K}_{i j} \boldsymbol{S}_{j}\right\rangle .
\end{aligned}
$$

In the classical limit and at vanishing temperature the quantum mechanical expectation value $\left\langle\boldsymbol{S}_{i} \times \mathbb{K}_{i j} \boldsymbol{S}_{j}\right\rangle$ can be factorized,

$$
\left\langle\boldsymbol{S}_{i} \times \mathbb{K}_{i j} \boldsymbol{S}_{j}\right\rangle \rightarrow\left\langle\boldsymbol{S}_{i}\right\rangle \times \mathbb{K}_{i j}\left\langle\boldsymbol{S}_{j}\right\rangle \equiv \boldsymbol{m}_{i} \times \mathbb{K}_{i j} \boldsymbol{m}_{j}
$$

Consequently the expectation value of the longitudinal spin current operator vanishes identically in this limit. This implies that for a generic magnetic insulator with a spin Hamiltonian of the form of Eq. 21) incoherent thermal or quantum fluctuations are a necessary prerequisite for the transport of actual magnetization. The local precession frequency reduces in the same limit to

$$
\begin{aligned}
\boldsymbol{\omega}_{i} & =-\boldsymbol{h}_{i}-\sum_{j} \hat{\boldsymbol{m}}_{i} \times\left(\hat{\boldsymbol{m}}_{i} \times \mathbb{K}_{i j} \boldsymbol{m}_{j}\right) \\
& =-\boldsymbol{h}_{i}+\sum_{j}\left[\mathbb{K}_{i j} \boldsymbol{m}_{j}-\hat{\boldsymbol{m}}_{i}\left(\hat{\boldsymbol{m}}_{i} \cdot \mathbb{K}_{i j} \boldsymbol{m}_{j}\right)\right] .
\end{aligned}
$$

The last term in Eq. (31) is proportional to $\hat{\boldsymbol{m}}_{i}$ and hence does not contribute to $\boldsymbol{\omega}_{i} \times \boldsymbol{m}_{i}$ so that we may write 
$\boldsymbol{\omega}_{i} \times \boldsymbol{m}_{i}=\left(-\boldsymbol{h}_{i}-\delta \boldsymbol{h}_{i}\right) \times \boldsymbol{m}_{i}$, where the renormalization of the magnetic field is given by

$$
\delta \boldsymbol{h}_{i}=-\sum_{j} \mathbb{K}_{i j} \boldsymbol{m}_{j} .
$$

Note that Eq. (32) can also be obtained by means of a simple mean-field decoupling of the spin Hamiltonian in Eq. 21).

\section{SPIN SUPERFLUIDITY AND PERSISTENT SPIN CURRENTS}

To illustrate the differences between translational and angular spin transport and how this affects the interpretation of spin supercurrents, it is instructive to consider simple model systems that can support translational and angular spin currents in equilibrium or in metastable states.

\section{A. Easy-plane ferromagnet}

Let us first consider an easy-plane ferromagnet described by the spin $S$ Heisenberg Hamiltonian

$$
\mathcal{H}^{\text {plane }}=-\frac{1}{2} \sum_{i j} J_{i j} \boldsymbol{S}_{i} \cdot \boldsymbol{S}_{j}+\frac{K}{2} \sum_{i} S_{i}^{z} S_{i}^{z}
$$

on a simple cubic lattice, with exchange coupling $J_{i j}=$ $J>0$ for nearest neighbors only, and an easyplane anisotropy $K>0$. This kind of systems has served in the literature as elementary example for spin

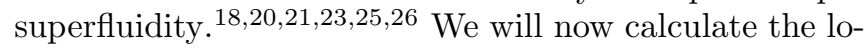
cal precession frequency 28 and the translational spin current 29 for this system to leading order in an $1 / S$ expansion. To facilitate this we expand the spin operators in a local basis defined by the instantaneous direction of the spin polarization $\hat{\boldsymbol{m}}_{i}(t)=\left\langle\boldsymbol{S}_{i}(t)\right\rangle /\left|\left\langle\boldsymbol{S}_{i}(t)\right\rangle\right|$ :

$$
\boldsymbol{S}_{i}=S_{i}^{\|} \hat{\boldsymbol{m}}_{i}+S_{i}^{(1)} \boldsymbol{e}_{i}^{(1)}+S_{i}^{(2)} \boldsymbol{e}_{i}^{(2)} .
$$

Here, $\boldsymbol{e}_{i}^{(1)}(t)$ and $\boldsymbol{e}_{i}^{(2)}(t)$ are unit vectors chosen such that $\left\{\boldsymbol{e}_{i}^{(1)}, \boldsymbol{e}_{i}^{(2)}, \hat{\boldsymbol{m}}_{i}\right\}$ form a right-handed basis at every lattice site. In this basis we may now bosonize the spin operators by means of a Holstein-Primakoff (HP) transformation,

$$
\begin{aligned}
S_{i}^{\|} & =S-a_{i}^{\dagger} a_{i}, \\
S_{i}^{(1)}+i S_{i}^{(2)} & =\sqrt{2 S} a_{i}+\mathcal{O}\left(S^{-1 / 2}\right),
\end{aligned}
$$

where the $a_{i}$ are canonical Bose operators. Especially note that since we self-consistently define the quantization axis as the direction of the local magnetization, $\hat{\boldsymbol{m}}_{i}(t)=\left\langle\boldsymbol{S}_{i}(t)\right\rangle /\left|\left\langle\boldsymbol{S}_{i}(t)\right\rangle\right|$, by definition the HP bosons can never condense. ${ }^{29}$ This is completely analogous to the fact that in the superfluid phase of interacting bosons the Bogoliubov quasi-particles, which are the Goldstone modes associated with the spontaneous breaking of the U(1)-symmetry in the superfluid state, do not condense provided the condensate wave-function is self-consistently defined via the solution of the GrossPitaevskii equation.

With the HP bosonization (35), we find that the leading order contributions to the precession frequency and the spin current are of order $S$. Explicitly, the local precession frequency 28 becomes

$$
\boldsymbol{\omega}_{i}=-\sum_{j} S J_{i j} \hat{\boldsymbol{m}}_{j}+S K\left(\hat{\boldsymbol{m}}_{i} \cdot \boldsymbol{e}_{z}\right) \boldsymbol{e}_{z} .
$$

To this order, the polarization equation of motion (2) recasts the Landau-Lifshitz equation of classical spin dynamics. Assuming that the magnetic texture $\hat{\boldsymbol{m}}_{i}=$ $\hat{\boldsymbol{m}}\left(\boldsymbol{R}_{i}\right)$ varies only slowly in space, we can take the continuum limit. The exchange contribution to the polarization equation of motion can then be identified with the divergence of the classical spin current,

$$
\sum_{j} S J_{i j} \hat{\boldsymbol{m}}_{j} \times \hat{\boldsymbol{m}}_{i} \rightarrow \sum_{\mu} \partial_{\mu} \boldsymbol{J}^{\mu},
$$

where the classical spin current is defined as

$$
\boldsymbol{J}^{\mu}=-S J a^{2} \hat{\boldsymbol{m}} \times \partial_{\mu} \hat{\boldsymbol{m}} .
$$

Here $\partial_{\mu}=\partial / \partial r^{\mu}$, and $a$ is the distance between nearest neighbors. An exact equilibrium solution for the polarization is then given by $\hat{\boldsymbol{m}}(\boldsymbol{r})=$ $\left[\boldsymbol{e}_{x} \cos \phi(\boldsymbol{r})+\boldsymbol{e}_{y} \sin \phi(\boldsymbol{r})\right]$, with a local phase satisfying $\nabla^{2} \phi(\boldsymbol{r})=0$. The $U(1)$ freedom associated with the choice of $\phi(\boldsymbol{r})$ lies at the heart of the concept of spin superfluidity. 20126 Out of equilibrium we can make the general ansatz

$$
\begin{aligned}
\hat{\boldsymbol{m}}(\boldsymbol{r}, t)= & \sqrt{1-\rho^{2}(\boldsymbol{r}, t)}\left[\boldsymbol{e}_{x} \cos \phi(\boldsymbol{r}, t)+\boldsymbol{e}_{y} \sin \phi(\boldsymbol{r}, t)\right] \\
& +\boldsymbol{e}_{z} \rho(\boldsymbol{r}, t)
\end{aligned}
$$

which is depicted graphically in Fig. 2. If the system is only slightly driven out of equilibrium, the equation of motion (2) for the spin polarization becomes to lowest nonvanishing order in deviations from equilibrium

$$
\begin{aligned}
\partial_{t} \phi & =S K \rho, \\
\partial_{t} \rho & =S J a^{2} \nabla^{2} \phi .
\end{aligned}
$$

The above Eqs. 40 are of the form of the Josephson equations of superconductivity; hence they are conventionally interpreted as describing a spin supercurrent carried by the magnetization texture.20|23 However, since this current does not correspond to translational transport of the local magnetization $\left|\boldsymbol{m}_{i}\right|=\left|\left\langle\boldsymbol{S}_{i}\right\rangle\right|$, this supercurrent is not equivalent to the superfluid transport of magnetic moments. In particular, a stationary angular supercurrent with $\nabla \phi=$ const is simply an inhomogeneous magnetic texture; while it can be viewed as a stationary transport of polarization, it does not correspond 


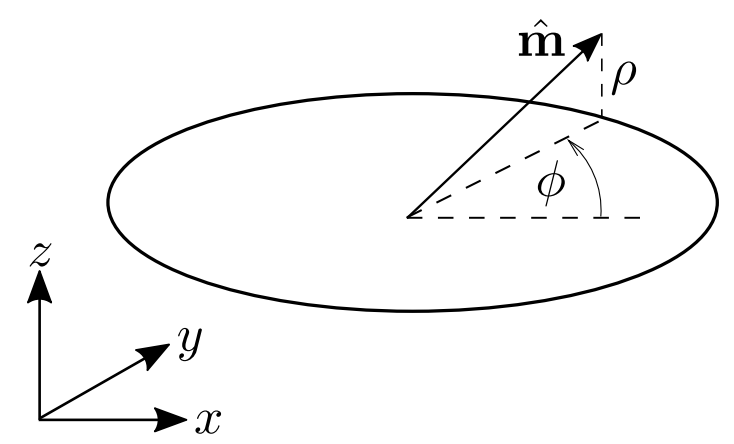

FIG. 2. Magnetic polarization in the easy-plane ferromagnet. $\rho$ denotes the deviation from the ground state with the magnetization lying in the $x$ - $y$-plane. Superfluid angular transport is possible due to the $U(1)$ freedom of the phase $\phi$ in this plane.

to any physical movement of magnetic moments which would generate an electrical field $\frac{17}{17}$

To evaluate the $\mathcal{O}(S)$ contribution to the translational spin current 29), we will assume for simplicity a slowly varying magnetic texture $\hat{\boldsymbol{m}}_{i}$ in a metastable superfluid state with $\rho \ll 1$. Applying the HP transformation (35) to the spin current 29 then yields

$$
\left\langle I_{i \rightarrow j}^{\|}\right\rangle=\operatorname{Im}\left[-2 S J_{i j}\left\langle a_{i}^{\dagger} a_{j}\right\rangle+\delta_{i j} S K\left\langle a_{i} a_{i}\right\rangle\right],
$$

which can to be evaluated to

$$
\left\langle I_{i \rightarrow j}^{\|}\right\rangle=\frac{1}{N a} \sum_{\boldsymbol{k}} v_{\boldsymbol{k}}^{\mu}\left[n_{\boldsymbol{k}}+\frac{1}{2}\left(1-\frac{E_{\boldsymbol{k}}}{A_{\boldsymbol{k}}}\right)\right],
$$

where $\boldsymbol{R}_{i}$ and $\boldsymbol{R}_{j}=\boldsymbol{R}_{i}+a \boldsymbol{e}_{\mu}$ are nearest neighbor lattice sites. Here $n_{\boldsymbol{k}}$ is the distribution functions of magnons with dispersion

$$
E_{k}=S \sqrt{\left(K+J_{k=0}-J_{k}\right)\left(J_{k=0}-J_{k}\right)}
$$

and velocity $v_{\boldsymbol{k}}^{\mu}=\partial E_{\boldsymbol{k}} / \partial k^{\mu}$, and the remaining coefficient of the quantum-mechanical zero-point fluctuations is

$$
A_{\boldsymbol{k}}=S\left(J_{\boldsymbol{k}=0}-J_{\boldsymbol{k}}\right)+S K / 2 .
$$

A detailed derivation of the Hamiltonian in the local basis and of the magnon dispersion 43 is relegated to the Appendix. In equilibrium the translational current 420 of course vanishes by symmetry, i.e., $\left\langle I_{i \rightarrow j}^{\|}\right\rangle=0$. Therefore there is no translational movement of magnetic moment in this superfluid spin state.

Lastly, let us note that the complete decoupling of the magnetic texture and the incoherent magnons is an artifact of the lowest order approximation in the $1 / S$ expansion, and of the assumption of a slowly varying texture. If one relaxes either of these approximations, there will be a coupling, resulting in a two-fluid description of

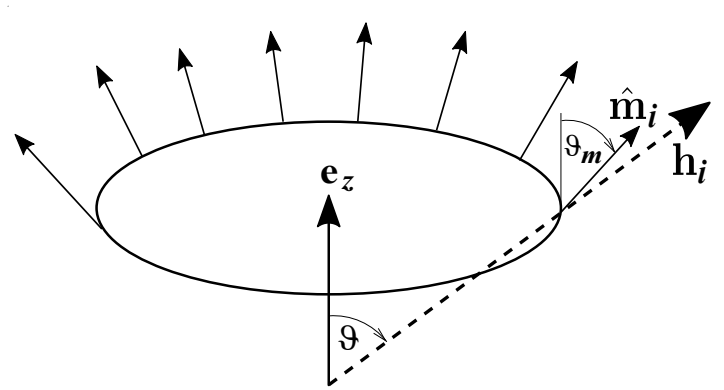

FIG. 3. Magnetization configuration of a ferromagnetic ring in a radial magnetic field. Due to the exchange field the angle $\vartheta_{m}$ between the magnetization and the $z$-axis is slightly smaller then the angle $\vartheta$ between the external field and the $z$-axis, see Ref. [4].

spin transport like the one discussed in [25]. We emphasize however that from the point of view we adopted, the superfluid does not arise due to the condensation of magnons. We rather consider the magnons as fluctuations on top of the superfluid ground state; these magnons cannot condense by definition.

\section{B. Heisenberg ring}

Next, let us consider a system where the translational spin current is finite even in equilibrium, i.e., there is a persistent translational spin current corresponding to the phyiscal movement of magnetic moments. Consider a ferromagnetic spin $S$ Heisenberg model in a radial inhomogeneous magnetic field with quantum mechanical Hamiltonian

$$
\mathcal{H}^{\mathrm{ring}}=-\frac{1}{2} \sum_{i j} J_{i j} \boldsymbol{S}_{i} \cdot \boldsymbol{S}_{j}-\sum_{i} \boldsymbol{h}_{i} \cdot \boldsymbol{S}_{i}
$$

where the sums are over the $N$ sites of the lattice of localized spins on the ring coupled by ferromagnetic exchange interactions $J_{i j}=J>0$ if $i$ and $j$ label nearest neighbors. $\boldsymbol{h}_{i}$ is a crown-shaped inhomogeneous magnetic field of the form

$$
\boldsymbol{h}_{i}=h\left[\sin \vartheta\left(\boldsymbol{e}_{x} \cos \varphi_{i}+\boldsymbol{e}_{y} \sin \varphi_{i}\right)+\boldsymbol{e}_{z} \cos \vartheta\right],
$$

where the angles $\varphi_{i}$ label the positions of the spins on the ring, as illustrated in Fig. 3. In Ref. [4] it has been shown that at finite temperature $T>0$ spin-wave excitations carry a persistent equilibrium spin current

$$
\left\langle I_{i \rightarrow i+1}^{\|}\right\rangle=\frac{1}{L} \sum_{n} \frac{v_{n}}{e^{\left(\epsilon_{n}+|\boldsymbol{h}|\right) / T}-1}
$$

circulating the ring. Here $L$ is the length of the ring, $v_{n}=\partial \epsilon_{n} / \partial k_{n}$ is the magnon velocity, and the magnon dispersion is $\epsilon_{n}=S J a^{2} k_{n}^{2}$, with lattice spacing $a$ and quantized wavevectors $k_{n}=\frac{2 \pi}{L}\left(n-\frac{\Omega}{2 \pi}\right) . \Omega$ is the solid 
angle traced out by the local magnetization direction $\hat{\boldsymbol{m}}_{i}$ on the unit sphere in order parameter space as it moves around the chain. This finite solid angle $\Omega$, i.e., the topology of the spin configuration on the ring, is responsible for the finiteness of the equilibrium current (47). The situation is completely analogous to persistent electrical currents in mesoscopic metal rings pierced by a magnetic flux. ${ }^{4}$ These electrical currents generate a magnetic dipole field; one of us has shown in Ref. 4 that the persistent spin current (47) similarly generates an electric dipole field. This can be understood as follows: $\frac{17}{17}$ A magnetic dipole moment $\boldsymbol{m}$ moving with velocity $\boldsymbol{v}$ generates a magnetic field $\boldsymbol{B}$ in its rest frame. Lorentztransforming back to the laboratory frame, we find that to lowest order in $\boldsymbol{v} / c$ (where $c$ is the speed of light) this magnetic field generates an electrical field $\boldsymbol{E}=-\frac{\boldsymbol{v}}{\boldsymbol{f}} \times \boldsymbol{B}$.

At zero temperature the equilibrium current (47) vanishes because there are no spin waves in the ferromagnetic ground state. However, Bruno and Dugaev ${ }^{7}$ pointed out that in this system the classical spin current $\boldsymbol{J}^{\mu}$ defined in Eq. (38) is finite and argued that therefore the system exhibits an equilibrium spin supercurrent even at $T=0$. While this interpretation is possible, we stress that this classical current is angular and not translational; hence it cannot be associated with the motion of magnetic dipoles, but should rather be considered as a renormalization of the external magnetic field ${ }^{5}$ To understand this, let us explicitly calculate the inhomogeneous magnetization configuration in the classical ground state of the Hamiltonian (45). Just as in the easy-plane ferromagnet, the equation of motion for the magnetic texture $\hat{\boldsymbol{m}}_{i}$ is to leading order in $1 / S$ the classical Landau Lifshitz equation

$$
\partial_{t} \hat{\boldsymbol{m}}_{i}=\hat{\boldsymbol{m}}_{i} \times\left(\boldsymbol{h}_{i}+\delta \boldsymbol{h}_{i}^{\mathrm{ex}}\right),
$$

where the exchange field is $\delta \boldsymbol{h}_{i}^{\text {ex }}=S \sum_{i j} J_{i j} \hat{\boldsymbol{m}}_{j}$. The equilibrium solution of the Landau-Lifshitz equation (48) is

$$
\hat{\boldsymbol{m}}_{i}=\sin \vartheta_{m}\left(\boldsymbol{e}_{x} \cos \varphi_{i}+\boldsymbol{e}_{y} \sin \varphi_{i}\right)+\boldsymbol{e}_{z} \cos \vartheta_{m},
$$

where the angle $\vartheta_{m}$ is slightly smaller than the angle $\vartheta$ between the magnetic field and the $z$-axis, as discussed in Ref. 4. The deviation of $\vartheta_{m}$ from $\vartheta$ is determined by the exchange field $\delta \boldsymbol{h}_{i}^{\text {ex }}$. As for the easy-plane ferromagnet discussed in the last section, the continuum limit of the exchange torque is the divergence of the classical spin current, $\delta \boldsymbol{h}_{i}^{\text {ex }} \times \hat{\boldsymbol{m}}_{i} \rightarrow \sum_{\mu} \partial_{\mu} \boldsymbol{J}^{\mu}$. For the classical ground state 49 , the classical spin current has a finite component $\propto \boldsymbol{e}_{z} \nabla \varphi$. However, as in the easy-plane ferromagnet, this finite current merely signals an inhomogeneous magnetization configuration and is not related to the physical transport of magnetization; hence it also will not generate any electrical field. If one would on the other hand incorrectly associate the classical spin current $\boldsymbol{J}^{\mu}$ with the stationary movement of physical dipoles, it would have to be accompanied by an electrical field. This would imply that a purely static inhomogeneous magne- tization configuration is always accompanied by an electric field, in contradiction with the elementary fact that in classical electromagnetism magnetostatics and electrostatics are completely decoupled.

Finally, let us also point out that although the classical ground state 49) of the Heisenberg ring is very similar to the classical ground state of the easy-plane ferromagnet discussed in the last section, it does not support spin superfluidity, i.e., angular spin supercurrents, because the phase $\varphi_{i}$ of the magnetization is pinned by the external magnetic field $\boldsymbol{h}_{i}$, Eq. 46.

\section{SUMMARY AND CONCLUSIONS}

We conclude that in the classical limit the angular spin current introduced by Sun and $\mathrm{Xic}^{8}$ can be absorbed into a renormalization of the external magnetic field which contributes to the torque acting on the magnetic moments. In equilibrium, this term does not describe any current of magnetic moments because the equilibrium configuration of the magnetization is such that the total torque on each moment vanishes. Translational transport of spins is described by the longitudinal spin current defined in Eq. (29), which is only finite due to thermal or quantum fluctuations. An example for a system exhibiting a finite longitudinal spin current in equilibrium is a mesoscopic Heisenberg ring in a crown-shaped magnetic field, as discussed in Ref. [4.

Our considerations imply that in equilibrium the classical spin current $\boldsymbol{J}^{\mu}$ defined in Eq. (38) does not describe any motion of magnetization. While it can be interpreted as a stationary current of magnetic polarization, such an interpretation is by no means mandatory since there is no phyiscal movement. In nonequilibrium on the other hand, the classical spin current $\boldsymbol{J}^{\mu}$ contributes to the angular spin current, i.e., to the precessional motion of the spins, and does transport spin polarization. In particular, this implies that spin superfluidity, which is based on the formal similarity of Eq. (40) with a mass supercurrent of superfluid bosons, ${ }_{226}^{26}$ does not correspond to the physical movement of magnetization, but of polarization. This means that in equilibrium, a superfluid spin state will not be accompanied by an electrical field, in contrast to a persistent translational spin current. ${ }^{4}$ This physical difference between the two types of spin transport persists also out of equilibrium: As already shown by Sun and Xie,$^{8}$ an angular spin current with finite $\boldsymbol{\omega} \times \hat{\boldsymbol{m}}$ will generate an electrical field $\boldsymbol{E}^{\omega} \propto 1 / r^{2}$ for large distance $r$ from the source, whereas the electrical field of translational spin currents decays as $\boldsymbol{E}^{\|} \propto 1 / r^{3}$.

We have also shown that spin superfluidity can be described entirely without referring to off-diagonal longrange order $\frac{30131}{1}$ and magnon condensation. This is achieved by quantizing the spins in a self-consistently defined frame of reference with the local $z$-axis pointing in the direction $\hat{\boldsymbol{m}}$ of the instantaneous magnetization. Magnons defined with respect to this reference frame 
can never condense or display off-diagonal long-range order, hence they are not superfluid. This is in agreement with the general proof of Kohn and Sherrington 31 that bosonic quasi-particles which are formed as bound states of particle-hole pairs of the underlying fermionic system (such as excitons or magnons) do not exhibit offdiagonal long-range order in coordinate space. Hence, the Bose-Einstein condensation of this type of bosons is not accompanied by superfluidity. Although the change in magnetic order in a magnetic insulator can be viewed as Bose-Einstein condensation of magnons, ${ }^{25|26| 32 \mid 33}$ the resulting state can always be characterized by magnons that exhibit neither off-diagonal long-range order nor superfluidity.

\section{ACKNOWLEDGMENTS}

This work was financially supported by the DFG via SFB/TRR49. Our ideas on spin transport were sharpened during a workshop on Quantum Spintronics: Spin Transport Through Quantum Magnetic Materials at the Spin Phenomena Interdisciplinary Center (SPICE) at the University of Mainz, Germany.

\section{APPENDIX: MAGNONS IN THE ROTATING REFERENCE FRAME}

This Appendix is devoted to the derivation of the dispersion (43) of the magnons in the local reference frame defined by the magnetic polarization $\hat{\boldsymbol{m}}_{i}(t)=$ $\left\langle\boldsymbol{S}_{i}(t)\right\rangle /\left|\left\langle\boldsymbol{S}_{i}(t)\right\rangle\right|$. We have already derived the general setup of a spin-wave expansion in this local and possibly time dependent reference frame in Ref. 29. The first step is to rotate the $z$-axis of the laboratory frame to the direction $\hat{\boldsymbol{m}}_{i}(t)$ of the local magnetization by means of an unitary transformation $\mathcal{U}(t)$ acting on the spins. The explicit form of $\mathcal{U}(t)$ is given in Ref. 29]. It then turns out that the rotated Hamiltonian $\tilde{\mathcal{H}}^{\text {plane }}=\mathcal{H}^{\text {plane }}+\mathcal{H}_{B}$ contains an additional Berry-phase term acting as a magnetic field due to the time dependence of $\hat{\boldsymbol{m}}_{i}(t)$,

$$
\mathcal{H}_{B}=-i \mathcal{U}^{\dagger} \partial_{t} \mathcal{U}=-\sum_{i} \boldsymbol{B}_{i} \cdot \boldsymbol{S}_{i}
$$

Writing the spin polarization as

$$
\hat{\boldsymbol{m}}_{i}=\sin \theta_{i}\left[\boldsymbol{e}_{x} \cos \phi_{i}+\boldsymbol{e}_{y} \sin \phi_{i}\right]+\boldsymbol{e}_{z} \cos \theta_{i}
$$

we can choose the transverse basis vectors as

$$
\begin{aligned}
& \boldsymbol{e}_{i}^{(1)}=-\boldsymbol{e}_{x} \sin \phi_{i}+\boldsymbol{e}_{y} \cos \phi_{i} \\
& \boldsymbol{e}_{i}^{(2)}=-\cos \theta_{i}\left[\boldsymbol{e}_{x} \cos \phi_{i}+\boldsymbol{e}_{y} \sin \phi_{i}\right]+\boldsymbol{e}_{z} \sin \theta_{i}
\end{aligned}
$$

Expanding the Berry-phase magnetic field in this basis, $\boldsymbol{B}_{i}=B_{i}^{(1)} \boldsymbol{e}_{i}^{(1)}+B_{i}^{(2)} \boldsymbol{e}_{i}^{(3)}+B_{i}^{\|} \hat{\boldsymbol{m}}_{i}$, we explicitly find

$$
\begin{aligned}
B_{i}^{(1)} & =\partial_{t} \theta_{i}=-\boldsymbol{e}_{i}^{(2)} \cdot \partial_{t} \hat{\boldsymbol{m}}_{i}, \\
B_{i}^{(2)} & =\sin \theta_{i} \partial_{t} \phi_{i}=\boldsymbol{e}_{i}^{(1)} \cdot \partial_{t} \hat{\boldsymbol{m}}_{i}, \\
B_{i}^{\|} & =\cos \theta_{i} \partial_{t} \phi_{i} .
\end{aligned}
$$

Applying the Holstein-Primakoff transformation 35 to the rotated Hamiltonian then yields

$$
\tilde{\mathcal{H}}^{\text {plane }}=E_{0}+\mathcal{H}_{1}+\mathcal{H}_{2}+\mathcal{O}\left(S^{1 / 2}\right) .
$$

Here the classical ground state energy is

$E_{0}=-\frac{S^{2}}{2} \sum_{i j} J_{i j} \hat{\boldsymbol{m}}_{i} \cdot \hat{\boldsymbol{m}}_{j}+\frac{S^{2}}{2} K \sum_{i}\left(\hat{\boldsymbol{m}}_{i} \cdot \boldsymbol{e}_{z}\right)^{2}-S \sum_{i} B_{i}^{\|}$

The term linear in the Bose operators can be written as

$$
\mathcal{H}_{1}=\sqrt{\frac{S}{2}} \sum_{i} a_{i}\left(\boldsymbol{e}_{i}^{(2)}+i \boldsymbol{e}_{i}^{(1)}\right) \cdot\left(\partial_{t} \hat{\boldsymbol{m}}_{i}-\boldsymbol{\omega}_{\boldsymbol{i}} \times \hat{\boldsymbol{m}}_{i}\right)+\text { h.c. },
$$

where $\boldsymbol{\omega}_{i}$ is the $\mathcal{O}(S)$ local precession frequency, Eq. 36 . Because the magnetic polarization satisfies the equation of motion $\partial_{t} \hat{\boldsymbol{m}}_{i}=\boldsymbol{\omega}_{i} \times \hat{\boldsymbol{m}}_{i}$ up to this order in $1 / S$, we conclude that $\mathcal{H}_{1}$ vanishes identically. Note that this implies that the Holstein-Primakoff bosons cannot condense, a statement which remains true to all orders in $1 / S$ due to the self-consistency of the basis. Up to this point the discussion is completely general and applies to all magnetic insulators.

To evaluate the quadratic part of the rotated Hamiltonian we will assume as in the main text that the magnetic texture varies only slowly in space and is only slightly out of equilibrium. Then we may approximate $\hat{\boldsymbol{m}}_{i} \cdot \hat{\boldsymbol{m}}_{i+1} \approx 1$, $\boldsymbol{e}_{i}^{(p)} \cdot \boldsymbol{e}_{i+1}^{\left(p^{\prime}\right)} \approx \delta^{p p^{\prime}}, \boldsymbol{e}_{i}^{(p)} \cdot \hat{\boldsymbol{m}}_{i+1} \approx 0$, with $p, p^{\prime} \in\{1,2\}$, and $\cos \theta_{i} \approx 0$. With this simplifications we immediately obtain

$$
\begin{aligned}
\mathcal{H}_{2}=\sum_{\boldsymbol{k}} & {\left[S\left(J_{\boldsymbol{k}=0}-J_{\boldsymbol{k}}+\frac{K}{2}\right) a_{\boldsymbol{k}}^{\dagger} a_{\boldsymbol{k}}\right.} \\
& \left.-S \frac{K}{4}\left(a_{\boldsymbol{k}} a_{-\boldsymbol{k}}+\text { h.c. }\right)+S \frac{K}{4}\right],
\end{aligned}
$$

which describes free magnons with the dispersion 43 .
1 E. I. Rashba, Spin currents in thermodynamic equilibrium: The challenge of discerning transport currents, Phys. Rev.
B 68, 241315(R) (2003). 
${ }^{2}$ E. I. Rashba, Spin currents, spin populations, and dielectric function of noncentrosymmetric semiconductors Phys. Rev. B 70, 161201 (2004).

3 E. I. Rashba, Spin Dynamics and Spin Transport, J. Supercond. 18, 137 (2005).

4 F. Schütz, M. Kollar, and P. Kopietz, Persistent Spin Currents in Mesoscopic Heisenberg Rings, Phys. Rev. Lett. 91, 017205 (2003).

${ }^{5}$ F. Schütz, P. Kopietz, and M. Kollar, What are spin currents in Heisenberg magnets?, Eur. Phys. J. B 41, 557 (2004).

6 D. Culcer, J. Sinova, N. A. Sinistyn, T. Jungwirth, A. H. MacDonald, and Q. Niu, Semiclassical Spin Transport in Spin-Orbit-Coupled Bands, Phys. Rev. Lett. 93, 046602 (2004).

7 P. Bruno and V. K. Dugaev, Equilibrium spin currents and the magnetoelectric effect in magnetic nanostructures, Phys. Rev. B 72, 241302(R) (2005).

${ }^{8}$ Q.-F. Sun and X. C. Xie, Definition of the spin current: The angular spin current and its physical consequences, Phys. Rev. B 72, 245305 (2005).

9 J. Shi, P. Zhang, D. Xiao, and Q. Niu, Proper Definition of Spin Current in Spin-Orbit Coupled Systems Phys. Rev. Lett. 96, 076604 (2006).

${ }^{10}$ P. Zhang, Z. Wang, J. Shi, D. Xiao, and Q. Niu, Theory of conserved spin current and its application to a twodimensional hole gas, Phys. Rev. B 77, 075304 (2008).

${ }^{11}$ I. G. Bostrem, Jun-ichiro Kishine, and A. S. Ovchinnikov, Theory of spin current in chiral helimagnets, Phys. Rev. 78, 064425 (2008).

12 I. V. Tokatly, Equilibrium Spin Currents: Non-Abelian Gauge Invariance and Color Diamagnetism in Condensed Matter, Phys. Rev. Lett. 101, 106601 (2008).

${ }^{13}$ N. Bray-Ali and Z. Nussinov, Conservation and persistence of spin currents and their relation to the Lieb-SchulzMattis twist operators, Phys. Rev. B 80, 012401 (2009).

${ }^{14}$ Z. An, F. Q. Liu, Y. Lin, and C. Liu, The universal definition of spin current, Sci. Rep. 2, 388 (2012).

15 B. Berche, E. Medina, and A. López, Spin superfluidity and spin-orbit gauge symmetry fixing, Europhys. Lett. 97, 67007 (2012)

${ }^{16}$ K. Nakata, K. A. van Hoogdalem, P. Simon, and D. Loss, Josephson and persistent spin currents in Bose-Einstein condensates of magnons, Phys. Rev. B 90, 144419 (2014). ${ }^{17}$ J. E. Hirsch, Overlooked contribution to the Hall effect in ferromagnetic metals, Phys. Rev. B 60, 14787 (1999).
18 J. Knig, M. Chr. Bønsager, and A. H. MacDonald, Dissipationless Spin Transport in Thin Film Ferromagnets, Phys. Rev. Lett. 87, 187202 (2001).

19 F. S. Nogueira, K.-H. Bennemann, Spin Josephson effect in ferromagnet/ferromagnet tunnel junctions, Europhys. Lett. 67, 620 (2004).

20 E. B. Sonin, Spin currents and spin superfluidity, Adv. Phys. 59, 181 (2010).

21 E. B. Sonin, Spin superfluidity, coherent spin precession, and magnon BEC, J. Low Temp. Phys. 171, 757 (2013).

22 H. Chen, A. D. Kent, A. H. MacDonald, and I. Sodemann, Nonlocal transport mediated by spin supercurrents, Phys. Rev. B 90, 220401(R) (2014).

23 S. Takei and Y. Tserkovnyak, Superfluid Spin Transport Through Easy-Plane Ferromagnetic Insulators, Phys. Rev. Lett. 112, 227201 (2014).

24 S. Takei, B. I. Halperin, A. Yacoby, and Y. Tserkovnyak, Superfluid spin transport through antiferromagnetic insulators, Phys. Rev. B 90, 094408 (2014).

${ }_{25}$ B. Flebus, S. A. Bender, Y. Tserkovnyak, and R. A. Duine, Two-Fluid Theory for Spin Superfluidity in Magnetic Insulators, Phys. Rev. Lett. 116, 117201 (2016).

${ }^{20}$ H. Chen and A. H. MacDonald, Spin-Superfluidity and Spin-Current Mediated Non-Local Transport, arXiv:1604.02429.

27 A. H. MacDonald, S. M. Girvin, and D. Yoshioka, $t / U$ expansion for the Hubbard model, Phys. Rev. B 37, 9753 (1988).

28 T. Moriya, Anisotropic Superexchange Interaction and Weak Ferromagnetism, Phys. Rev. 120, 91 (1960).

${ }^{29}$ A. Rückriegel, A. Kreisel, and P. Kopietz, Time-dependent spin-wave theory, Phys. Rev. B 85, 054422 (2012).

so C. N. Yang, Concept of Off-Diagonal Long-Range Order and the Quantum Phases of Liquid He and of Superconductors, Rev. Mod. Phys. 34, 694 (1962).

${ }_{31}$ W. Kohn and D. Sherrington, Two Kinds of Bosons and Bose Condensates, Rev. Mod. Phys. 42, 1 (1970).

32 S.O. Demokritov, V.E. Demidov, O. Dzyapko, G.A. Melkov, A.A. Serga, B. Hillebrands, and A.N. Slavin, BoseEinstein condensation of quasi-equilibrium magnons at room temperature under pumping, Nature (London) 443, 430 (2006).

33 A. Rückriegel and P. Kopietz, Rayleigh-Jeans Condensation of Pumped Magnons in Thin-Film Ferromagnets. Phys. Rev. Lett. 115157203 (2015). 